\title{
Echoes of Asymptotic Silence in Causal Set Quantum Gravity
}

\author{
Astrid Eichhorn, ${ }^{1, *}$ Sebastian Mizera, ${ }^{2,3}$ and Sumati Surya ${ }^{4}$ \\ ${ }^{1}$ Institut für Theoretische Physik, Universität Heidelberg, \\ Philosophenweg 16, 69120 Heidelberg, Germany \\ ${ }^{2}$ Perimeter Institute for Theoretical Physics, Waterloo, Ontario N2L 2Y5, Canada \\ ${ }^{3}$ Department of Physics 83 Astronomy, University of Waterloo, Waterloo, Ontario N2L 3G1, Canada \\ ${ }^{4}$ Raman Research Institute, C.V. Raman Avenue, \\ Sadashivnagar, Bangalore 560 080, India
}

\begin{abstract}
We explore the idea of asymptotic silence in causal set theory and find that causal sets approximated by continuum spacetimes exhibit behavior akin to asymptotic silence. We make use of an intrinsic definition of spatial distance between causal set elements in the discrete analogue of a spatial hypersurface. Using numerical simulations for causal sets approximated by $D=2,3$ and 4 dimensional Minkowski spacetime, we show that while the discrete distance rapidly converges to the continuum distance at a scale roughly an order of magnitude larger than the discreteness scale, it is significantly larger on small scales. This allows us to define an effective dimension which exhibits dimensional reduction in the ultraviolet, while monotonically increasing to the continuum dimension with increasing continuum distance. We interpret these findings as manifestations of asymptotic silence in causal set theory.
\end{abstract}

\section{INTRODUCTION}

Several approaches to quantum gravity exhibit an ultraviolet reduction of the spectral dimension from four to (close to) two dimensions, where the spectral dimension is defined via a Riemannian diffusion process. These models include Dynamical Triangulations [1-7], asymptotically safe gravity [8 12], Hořava-Lifshitz gravity [13, 14], and several other models [15-21]. It was argued in [22 26] that this scale dependent dimension is related to the phenomenon of "asymptotic silence" in quantum spacetime. Asymptotic silence describes the "narrowing" or sharp focusing of lightcones near the Planck scale, leading to a decoupling of seemingly nearby worldlines. Near the singularities of a classical spacetime for example, asymptotic silence leads to a Kasner-like behavior of the metric, which exhibits dimensional reduction [27, 28]. Near the space-like singularity communication between different worldlines is prohibited.

In a quantum spacetime, properties such as the dimensionality can become scale-dependent. In particular, appropriately defined dimensional estimators can change as one "zooms in" to smaller scales, while they converge to their continuum values at larger scales. From a more fundamental point of view, one might even expect the standard manifold structure of spacetime to break down in the quantum regime.

Since the diffusion process that determines the spectral dimension in all the quantum gravity approaches mentioned above is Riemannian, relating it to a Lorentzian $1+1$ dimensional spacetime is far from obvious. Thus the connection between dimensional reduction and asymptotic silence is an indirect one. On the other hand, it may be possible to probe the quantum properties of the causal structure more directly in a manifestly Lorentzian approach like causal set theory (CST).

In CST the spacetime continuum is replaced by a fundamentally discrete structure, which is a locally finite partially ordered set. Spacetime discreteness is implemented by requiring that any spacetime region of finite volume in the continuum contains a finite number of fundamental

*Electronic address: a.eichhorn@thphys.uni-heidelberg.de 
spacetime "atoms" and that this number, on average, is the continuum spacetime volume with respect to the physical discreteness scale.

In [29] it was shown that results of numerical simulations of diffusion on causal sets show an increase of the spectral dimension on short scales ${ }^{1}$ which was interpreted as a possible signature of asymptotic silence in [31. The spectral dimension is obtained from a diffusion process in a fictitious, external time variable $\sigma$, and measures the return probability of the corresponding random walker to its starting point. A generalization introduced in [29] considers two causal random walkers with the spectral dimension given by

$$
d_{s}(\sigma)=-2 \frac{d \ln P(\sigma)}{d \ln \sigma}
$$

where $P(\sigma)$ is their meeting probability after time $\sigma$. A decrease in the meeting probability would therefore lead to an increase in the spectral dimension. This is what one might expect to happen in an asymptotically silent spacetime in which the worldlines are further apart because of a narrowing of the lightcones. Thus, dimensional increase could be attributed to asymptotic silence, as advocated in [31].

In this work we will explore this intriguing suggestion more directly. As stated in the usual way, the notion of asymptotic silence requires a foliation of spacetime by spacelike hypersurfaces since it refers to the decoupling of spatially "nearby" points. On the other hand, causal set quantum gravity is an intrinsically covariant or spacetime approach to quantum gravity and the notion of a foliation is ill defined in a causal set. It is therefore not immediately obvious how to translate what it means for a light cone to "narrow" as a function of time in this setting.

Instead, an "echo" of asymptotic silence in causal set theory comes unexpectedly from a calculation of causal set homology [32, 33]. Starting from an inextendible antichain $A$, which is the discrete analogue of a Cauchy hypersurface (for details on causal set concepts and terminology, see Sec. II), a family of homology groups was constructed as a function of $n \in \mathbb{N}$, which converges to give the continuum homology for large enough $n$, but not for smaller $n$. Here $n$ is a dimensionless measure of scale with small $n$ corresponding to the ultraviolet (UV) or discreteness scale and large $n$ to the continuum. In particular, for small $n$ there are a large number of spatially disconnected regions or "islands" in the underlying causal set, even for a connected spacetime. As $n$ increases, these islands join together and eventually converge to a single connected region. The existence of these islands is similar to the enhanced separation between neighbouring worldlines in the UV which characterise asymptotic silence. Indeed, as we will demonstrate, a similar construction can be used to define asymptotic silence in causal set theory.

In Section II we begin with a brief review of CST elucidating some of its key features which distinguish it from other approaches to quantum gravity. In Section III we give a definition of spatial distance $\delta$ in the discrete analogue of a Cauchy hypersurface and use it to show asymptotic silence in CST. We argue that $\delta$ is always greater than the (normalised) continuum proper distance $\mathbf{d}_{p}$ between the elements and that because of fluctuations arising from randomness, the difference in $\delta$ and $\mathbf{d}_{p}$ grows as $\mathbf{d}_{p}$ decreases.

This increased distance in the ultraviolet can be interpreted as a manifestation of asymptotic silence. We present results from numerical simulations for causal sets that are approximated by

\footnotetext{
${ }^{1}$ In [30, a nonlocal d'Alembertian was used to extract a spectral dimension which showed an initial dimensional increase towards the UV and a subsequent dimensional reduction at even smaller scales. Thus the results from numerical simulations 29] appear to be in contradiction with these analytical results. However the nonlocal d'Alembertian used in [30] is a fully continuum version of the causal set d'Alembertian. Its use in the far UV cannot therefore accurately reflect the effect of causal set discreteness. On the other hand, away from this scale the dimensional increase matches that of [29.
} 
$D=2,3$ and 4 Minkowski spacetime which strongly support this argument. Interestingly, we find that the approach to the continuum occurs at roughly the same length scale in all dimensions, $\sim 10 \times l_{c}$, where $l_{c}$ is the discreteness scale. We use $\delta$ and $\mathbf{d}_{p}$ to define an "effective dimension" $D_{\text {eff }}$ which exhibits dimensional reduction in the ultraviolet.

It is important to point out that the discreteness scale $l_{c}$ defines what we refer to as the ultraviolet scale for causal sets in this context and is distinct from the deep quantum regime characterised by the Planck scale $l_{p}$. This distinction is important in CST since the continuum need not play any role near $l_{p}$. Indeed, the full quantum theory of causal sets must include those that are not manifoldlike. Manifoldlike properties would only emerge after coarse graining of the causal set, at scales $l_{c} \gg l_{p}$. The phenomena we describe in this paper are therefore due to the traces of discreteness which are still present in the continuum approximation, at scales $\sim l_{c}$. Indeed, in this sense causal sets are quantum even without quantum interference, since the causal set underlying a given continuum spacetime carries a discreteness scale. Thus, causal sets differ from the continuum already at the kinematical level. The effect of this discreteness can lead to non-classical behaviour of test particles, as in the case of swerves [34, 35] or the scalar field Green's function [36]. From this perspective it is therefore reasonable to ask if causal sets exhibit traces of asymptotic silence even without probing the deep quantum regime.

\section{AN OVERVIEW OF CST}

The causal-set approach to quantum gravity is built on two fundamental principles: causality and discreteness [37] (for reviews see, e.g., 38-41]). The former is motivated by powerful theorems in Lorentzian geometry which state that under very weak causality conditions the causal structure poset gives the conformal geometry of the spacetime and also its topology and dimension [42, 43. The motivation for the latter comes from a variety of sources, such as the suggestion for the existence of a cut-off in the description of black hole entropy. This leads to a minimalistic mathematical structure for quantum spacetime, which is that of a locally finite partially ordered set or causal set $C$ defined as follows.

A causal set $C$ is a countable collection of elements which are partially ordered via a relation $\prec$ which is acyclic (if $x \prec y$ and $y \prec z \Rightarrow x=y$ ), transitive, $\forall x, y, z \in C: x \prec y, y \prec z \Rightarrow x \prec z$, and locally finite $(\forall x, y \in C: \operatorname{card}(\{z \in C: x \prec z \prec y\})<\infty)$.

Importantly, the requirement of local finiteness ensures that finite spacetime volumes in the continuum approximation contain a finite number of causal set elements, thus ensuring a fundamental discreteness. The continuum spacetime arises as an approximation of the underlying causal set with the order relation $\prec$ corresponding to the continuum causal order and such that the number of elements in a given region corresponds to the spacetime volume in units of a fundamental minimum volume. The approximation is implemented via a Poisson process (see [38-41]) so that the number of elements is equal to the spacetime volume on average.

It is this randomness that gives rise to interesting phenomenology [35, 45, 54]. In particular it ensures that despite discreteness, Lorentz invariance is not violated [55, 56], but instead leads to non-locality.

CST differs crucially from other discrete approaches to quantum gravity in that the discreteness is assumed to be physical, rather than a mathematical tool for regularising the theory. For instance in (Causal) Dynamical Triangulations [57 60], physics can only emerge in the continuum limit since the discretisation is introduced as an unphysical regulator. In CST, on the contrary, since the discreteness is physical, the continuum limit is not. Thus one refers to the continuum approximation rather than the continuum limit.

Causal sets come in two distinct types: Those which are approximated by $D$ dimensional 
spacetimes, and those which are not. The latter dominate the set of all causal sets entropically [61, and hence an important question in CST is whether a suitable dynamics can be found that will suppress the entropy in favour of manifoldlike causal sets in the appropriate limit (see [44, 62, 66. for more discussion on causal set dynamics). Since CST is a quantum theory of all causal sets, one does not expect those that are manifoldlike to play a significant role in the deep quantum regime; the continuum approximation becomes relevant only at larger scales $l_{c} \gg l_{p}$. Nevertheless, the remnant of the quantum nature of the causal set manifests itself near the discreteness scale $\sim l_{c}$, and it is this region that we explore ${ }^{2}$.

\section{ASYMPTOTIC SILENCE IN CST}

An antichain in $C$ is a set of unrelated elements and is the analogue of a set of spacelike related elements in a spacetime. The analogue of a Cauchy hypersurface in a globally hyperbolic spacetime is an inextendible antichain $A \subset C$, which is an antichain $A$ such that all elements in $C$ that are not in $A$ belong to its past or future, where for any set $S \subset C$

$$
\operatorname{Future}(S) \equiv\{e \in C \mid \exists s \in S, s \prec e\}, \quad \operatorname{Past}(S) \equiv\{e \in C \mid \exists s \in S, s \succ e\} .
$$

Inextendibility of $A$ thus ensures that any other element in $C$ is either to the past or the future of an element in $A$, so that $C$ can be divided into the non-overlapping regions $C=A \sqcup \operatorname{Future}(A) \sqcup$ $\operatorname{Past}(A)$.

$A$ itself contains no information, except its cardinality. Nevertheless, as explored in [32, 33] it can borrow structure from Future $(A) \subset C$ by "evolving" $A$ to a future neighbourhood. For every $e \in \operatorname{Future}(A)$, the cardinality

$$
v(e)=\operatorname{card}(\operatorname{Past}(e) \cap(A \cup \operatorname{Future}(A)))
$$

can be used to foliate Future $(A)$ by constant $v$ "slices", cf. Fig. 1. Though these slices are themselves antichains, they need not be inextendible, but this will not matter to the analysis. A thickened antichain is defined as

$$
T_{v}(A) \equiv\{e \in \operatorname{Future}(A) \mid v(e) \leq v\}
$$

$T_{v}(A)$ has more structure than $A$ itself and hence the potential to encode continuum geometrical and topological information for large enough $v$, see [67]. Conversely, for small $v$, one expects the continuum approximation to break down.

In [32, 33] an order theoretic construction capturing the spatial homology of a manifold-like causal set was given. The homology was seen to be a function of $v$ but stabilised after a certain critical value. The continuum topology is therefore emergent at large $v$, whereas discreteness manifests itself at small $v \sim V_{c}$. For small $v$ the simplicial complex has several disconnected "islands" leading to a large zeroth betti number even when the continuum is connected. As $v$ increases, these islands merge into a single connected component.

Finding a connection between stable homology and asymptotic silence is not obvious, but it provides an important clue. In particular, the discrete homology for a given $v$ is constructed from the causal structure of the elements in the thickening to the original antichain. This suggests a definition of a spatial distance on the inextendible antichain. For small $v$ the disconnected islands

2 Because of Lorentz invariance it is important to remember that the discreteness scale is characterised by the spacetime volume $V_{c}=l_{c}^{D}$ rather than a length scale. 


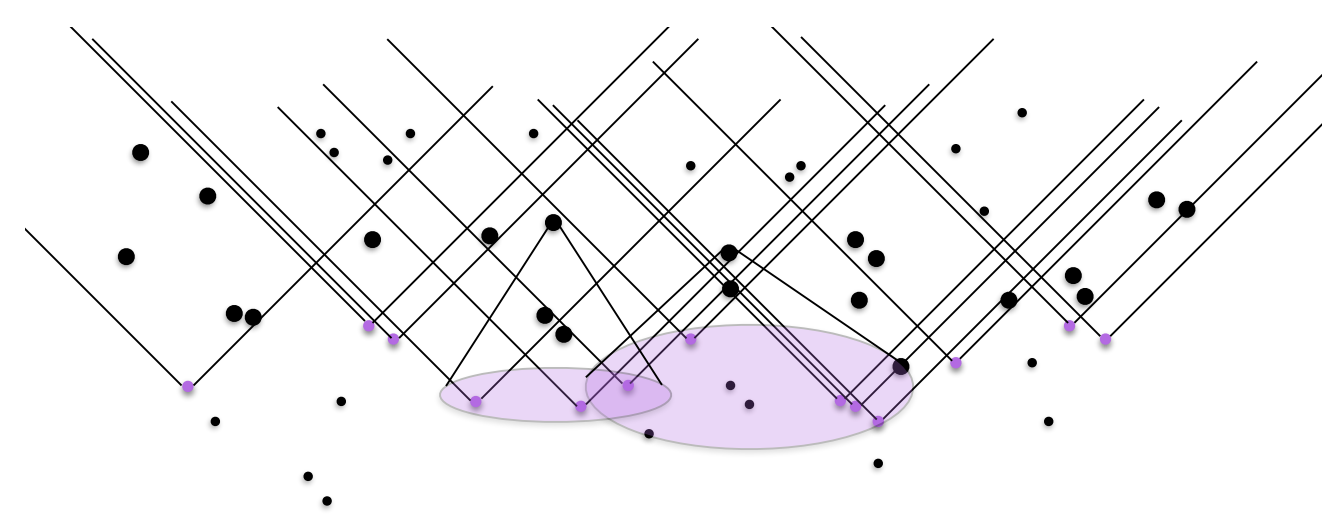

FIG. 1: We sketch an inextendible antichain (purple dots) in a sprinkling into two-dimensional Minkowski spacetime. Elements in a thickening with $v=5$ are shown as fat dots, and selected past and future lightcones are indicated.

suggest a decoupling of elements in the causal set that are nevertheless spatially close in the continuum. Exploiting this connection gives us a useful definition of asymptotic silence compatible with causal set discreteness.

We look to the continuum for guidance on how to proceed. In $D$-dimensional Minkowski spacetime, consider the events $p=\left(0, \vec{x}_{1}\right)$ and $q=\left(0, \vec{x}_{2}\right)$. Their proper distance $d_{p}=\left|\vec{x}_{1}-\vec{x}_{2}\right|$ can be related to a spacetime volume as follows. Without loss of generality, let the origin $(0, \overrightarrow{0})$ lie at the midpoint of $p$ and $q$ in the $t=0$ slice. The chronological past of the event $r=\left(d_{p} / 2, \overrightarrow{0}\right)$ does not include $p, q$, but its closure (which in this case is equal to its causal past) does. The past volume of $r$ up to this slice is given by

$$
V=\zeta_{D} d_{p}^{D}
$$

where

$$
\zeta_{D}=\frac{\pi^{(D-1) / 2}}{2^{D} D \Gamma\left(\frac{D+1}{2}\right)} .
$$

In Minkowski spacetime $d_{p}$ is a distance function on the $t=0$ slice, since it naturally satisfies the triangle inequality

$$
d_{p}(p, q) \leq d_{p}(p, s)+d_{p}(q, s)
$$

for all $p, q, s$ in the $t=0$ hypersurface. Indeed, this reproduces exactly the distance on the $t=0$ slice.

Casting the distance into this form makes it easier to construct the causal set analogue. For a given pair of elements $p, q \in A$, let $r \succ p, q$ such that $\operatorname{Past}(r) \ni p, q$. Let $n(r)=\operatorname{Past}(r) \cap \operatorname{Future}(A)$ and let $n$ denote the infimum of $n(r)$ over all such $r$. We then define the discrete causal set spatial distance as

$$
\delta=\left(\frac{n}{\zeta_{D}}\right)^{\frac{1}{D}} .
$$

How are we to compare $d_{p}$ which is dimensionful to the dimensionless $\delta$ ? For a causal set that is approximated by $D$-dimensional Minkowski spacetime it is natural to cast the proper distance in the continuum in units of the spacetime cut-off $V_{c}$,

$$
\mathbf{d}_{p} \equiv\left(\frac{V}{\zeta_{D} V_{c}}\right)^{\frac{1}{D}},
$$




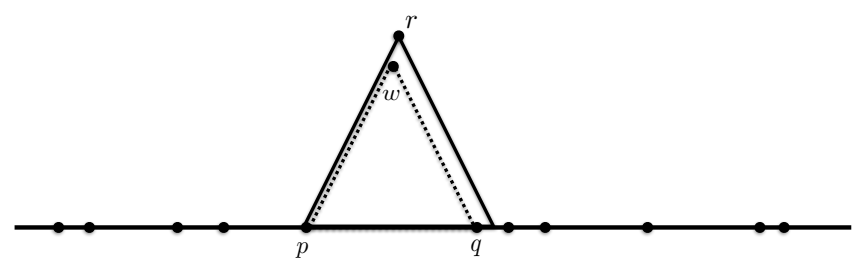

FIG. 2: An illustration showing why $\mathbf{d}_{p}<\delta$ at small scales. For two elements on the $t=0$ slice, $\mathbf{d}_{p}$ is obtained from the past volume of the spacetime event $w$. In the causal set the probability for an element to lie on $w$ is zero, and hence $\delta$ must be defined using the past of some $r \in C$ with $r \succ w$ which is larger.

in order to facilitate the comparison with $\delta$.

If we make the unjustified assumption that the antichain $A$ lies exactly on the $t=0$ slice, we would find that $\delta$ is strictly larger than $\mathbf{d}_{p}$ since the probability of $r$ being to the causal but not the chronological future of both $p$ and $q$ is zero. In other words, since null surfaces are a set of measure zero the probability that a pair of elements in a causal set are null related is zero. Thus $\operatorname{Past}(r) \cap \operatorname{Future}(A)$ is always slightly larger than $\operatorname{Past}((d / 2, \overrightarrow{0})) \cap \operatorname{Future}(A)$ in the continuum (see Fig. 2 for an illustration).

Moreover, at small scales, the relative fluctuations in the number of elements in a given spacetime region is larger, and hence the overestimation is substantially larger. As $n$ increases, it is reduced so that $\delta$ converges to $\mathbf{d}_{p}$. In the continuum, $\mathbf{d}_{p}$ translates into the shortest time taken for the worldlines of two observers starting at $p$ and $q$ to meet, with respect to the discreteness scale. Since $\delta>\mathbf{d}_{p}$, so too is the "time" taken for them to meet in the underlying causal set. Thus observers are further away from each other in the causal set than they would be in the continuum. This then is a direct manifestation of asymptotic silence.

Of course, the assumption that there exists such a flat antichain $A$ is incorrect and the comparisons must be made more carefully. Given a sprinkling of $C$ into a spacetime $(M, g)$ with compact spatial hypersurfaces, consider any inextendible antichain $A \subset C$. The proper distance $d_{p}$ of any pair $p, q \in A$ can be calculated from the embedding and expressed in terms of the cut-off $l_{c}$. However, its relationship to the volume $V$ of the past of an event $r \succ p, q$ is no longer as simple as Eqn. (5). To begin with, every $A$ lies in an uncountable infinity of spatial hypersurfaces $\{\Sigma\}$ and the choice of $\Sigma$ determines $V$. In particular the factor replacing $\zeta_{D}$ in Eqn. (5) will depend on the induced spatial geometry on $\Sigma$ as well as the location of $p, q \in \Sigma$. This information can then be used to calculate the discrete distance $\delta$ using Eqn. (8). Again, randomness ensures that $\delta>\mathbf{d}_{p}$, which translates into asymptotic silence.

To prove this more rigorously requires more extensive arguments, along the lines of [32, 33. In this work we will resort to the easier task of performing numerical simulations to support this claim. We obtain causal sets from a Poisson sprinkling into regions of $D=2,3$ and 4 dimensional Minkowski spacetime. Because of the calculational complications that arise in considering an arbitrary antichain $A$ we pick the region to be a $D$ dimensional "box" or hypercube, with the intial and final hypersurfaces corresponding to $t=0$ and $t=L$, respectively. Moreover, we choose $A$ to be the past-most inextendible antichain, so that it is reasonably well approximated by the $t=0$ slice. This then limits the systematic errors in the calculation. These errors come from our use of $\zeta_{D}$ as defined in Eqn. (6) even though the elements in $A$ do not strictly lie in the $t=0$ slice. By doing so, $\delta$ is sometimes a little underestimated. Defining

$$
\Delta \equiv \frac{\delta-\mathbf{d}_{p}}{\mathbf{d}_{p}}
$$

as a measure of asymptotic silence, we see that it becomes slightly negative for some datapoints 

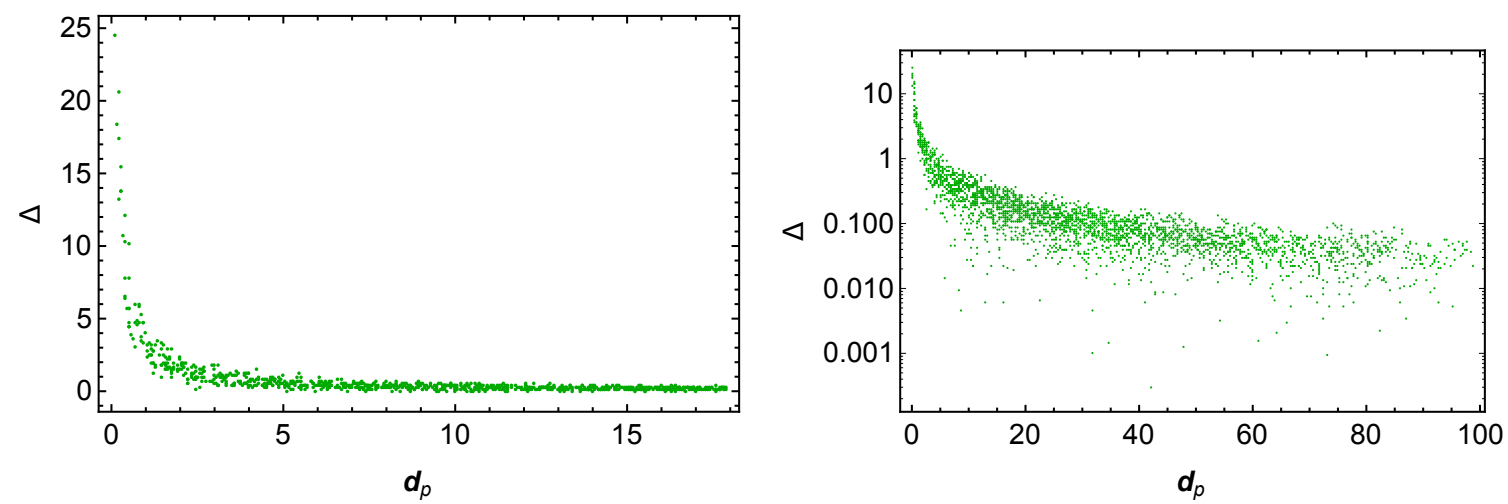

FIG. 3: We show $\Delta$ as a function of the dimensionless spatial distance $\mathbf{d}_{p}$ for $10^{5}$ elements for a causal set approximated by $1+1$ dimensional Minkowski spacetime, and for 100 trials. The logarithmic plot on the right shows the slow approach to the exact infrared limit, whereas the linear plot highlights that asymptotic silence does not persist beyond $\mathbf{d}_{p} \approx 10$.
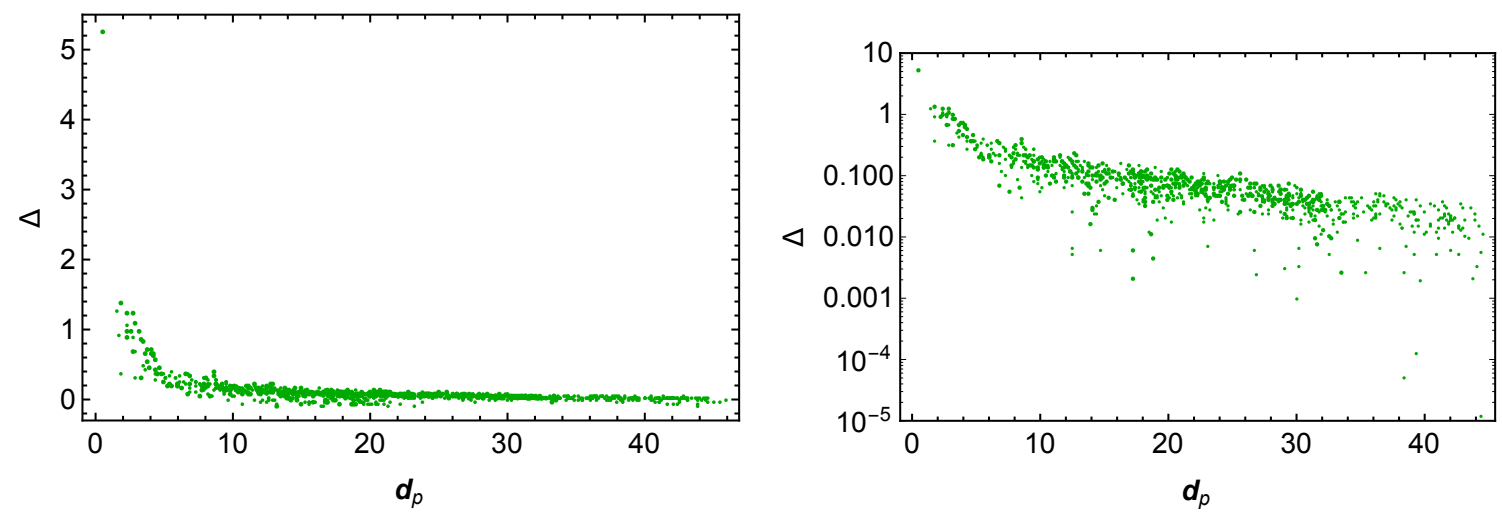

FIG. 4: We show $\Delta$ as a function of the dimensionless spatial distance $\mathbf{d}_{p}$ for a $10^{6}$ element causal set approximated by $2+1$ dimensional Minkowski spacetime.

even for large distances when there is better agreement with the continuum.

The simulation is performed by picking an element $e \in A$ at random and finding its causal set distance $\delta(e)$ to all other elements in $A$. The proper distance $\mathbf{d}_{p}$ can simultaneously be calculated from the embedding coordinates of $C$ into $D$ dimensional Minkowski spacetime. Ideally a good comparison is possible only if the length of the sprinkling box is the same in all dimensions. Starting with a box of size $100^{2}$ in $D=2$ this translates into $N=10^{6}$ and $N=10^{8}$ in $D=3$, 4 . These however need 64 and $6400 \mathrm{GBs}$ of RAM, respectively ${ }^{3}$. While the former is possible to achieve on modern machines, the latter is not. We will therefore content ourselves with the requirement that the box is large enough to begin to see emergent continuum behaviour. Our simulations are done for $N=10,000$ elements in $D=2, N=100,000$ elements for $D=3$ and $N=400,000$ elements for $D=4$. Because of the ease of simulation in $D=2$, we perform 100 trials which gives us independent data that can be plotted on the same graph as shown in Figure 3 . Our simulations were done using the Causal Set Cactus package [70].

In Figs. 3, 4 and 5, we see that $\Delta$ drops sharply down to near zero at a continuum distance $d_{p} \sim 10 l_{c}$ in all cases. Thus, as it should be, the causal set is well-approximated by the continuum

\footnotetext{
${ }^{3}$ For a causal set the number of possible relations is $\left(\begin{array}{c}N \\ 2\end{array}\right)$, which tranlates into $\sim N^{2} / 16$ GB of RAM.
} 

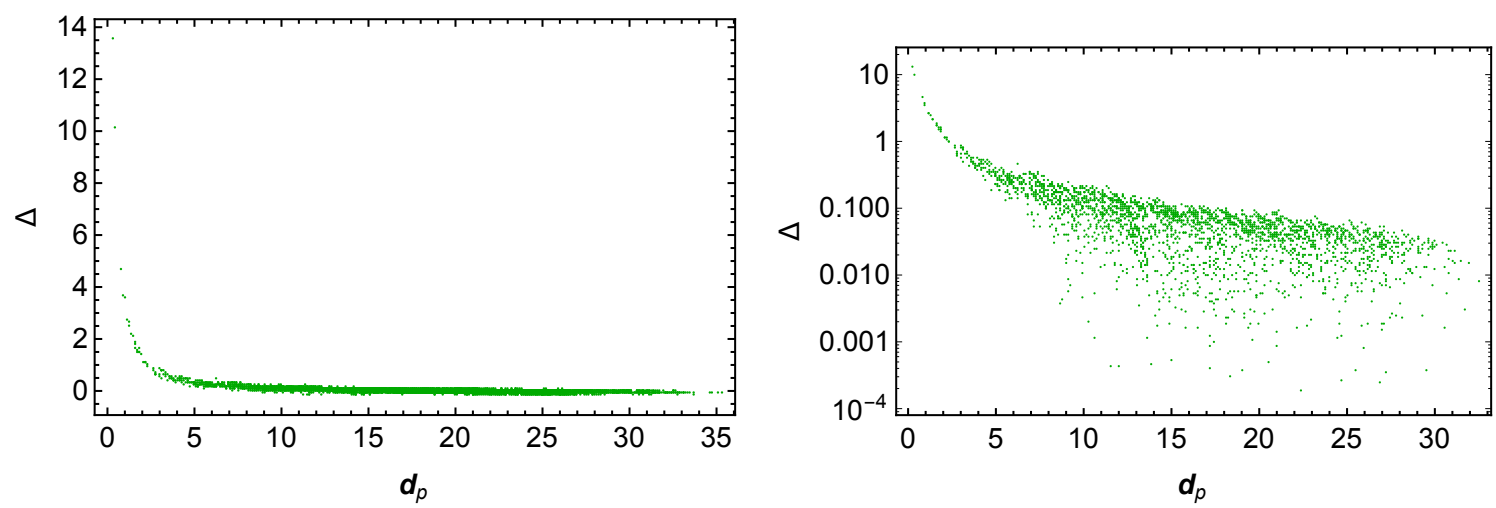

FIG. 5: We show $\Delta$ as a function of the dimensionless spatial distance $\mathbf{d}_{p}$ for a $4 \cdot 10^{6}$ element element causal set approximated by $3+1$ dimensional Minkowski spacetime.

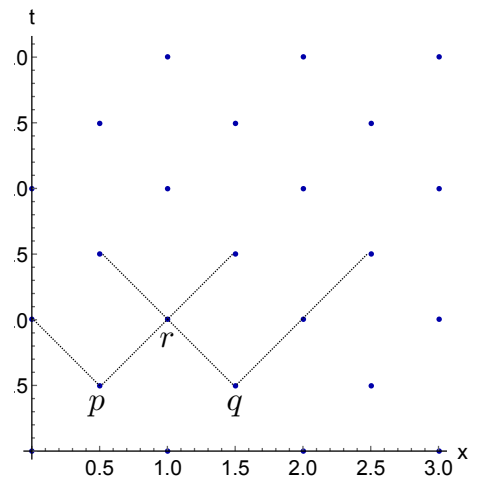

FIG. 6: We show a regular lightcone lattice in $1+1$ dimensions. The points $p, q$ and $r$ to illustrate that asymptotic silence does not occur.

manifold at scales larger than $10 l_{c}$, beyond which the discrete and the continuum distance merge. Around $d_{p} \simeq 10 l_{c}$, the approach of $\Delta$ to zero slows down significantly; thus small remnants of the discreteness remain. For smaller $\mathbf{d}_{p}, \Delta$ becomes fairly large, in particular even significantly larger than one, thus supporting the hypothesis of asymptotic silence.

To distinguish causal set discreteness from that of a regular lattice, it is instructive to consider a lightcone lattice in two dimensions, cf. Fig. 6. In contrast with the causal set case, the lattice breaks Lorentz invariance by introducing a preferred time foliation. Because of this, for every pair of points $p$ and $q$ on the same time slice, there is a unique point $r$, which lies exactly on the intersection of the lightcones of $p$ and $q$, cf. Fig. 6. Even if the lattice were tailored so that $\delta>\mathbf{d}_{p}$, the offset would be constant with $\mathbf{d}_{p}$. Hence, calculating the distance measure $\tilde{\delta}$ gives the same answer as in the continuum. We conclude that a deviation of $\delta$ from $\mathbf{d}_{p}$ is not a generic artifact of discretisation. Instead we attribute the observation of $\Delta>0$ to the randomness associated with causal set discreteness.

We can now extract an effective dimension $D_{\text {eff }}$ by comparing the volumes arising from $\delta$ and $\mathbf{d}_{p}$,

$$
(\delta)^{D_{\mathrm{eff}}}=\left(\mathbf{d}_{p}\right)^{D}
$$

The right hand side of the expression is the continuum spacetime volume of a box with sides of 


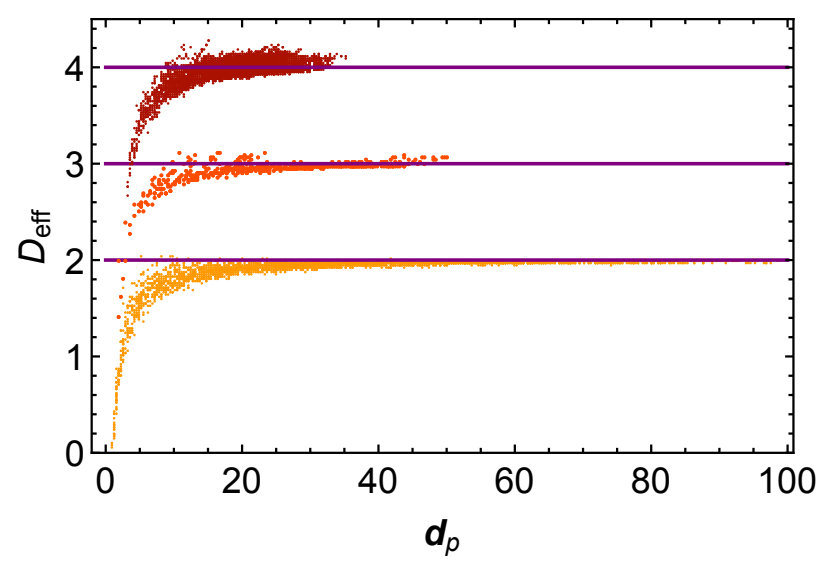

FIG. 7: We show the effective dimension $D_{\text {eff }}$ as a function of $\mathbf{d}_{p}$ for causal sets approximated by $D=2$ (orange dots), $D=3$ (red dots) and $D=4$ (dark red dots) Minkowski spacetimes. Each case exhibits dimensional reduction at small scales and approaches the continuum dimension at large scales. We have excluded points with $\mathbf{d}_{p}<l_{c}$.

length $\delta$ in terms of the cut-off scale $V_{c}$. $D_{\text {eff }}$ is thus defined by asking what the dimension of a hypercube of side $\delta$ should be if it is to give the same continuum volume as that obtained from $\mathbf{d}_{p}$. This comparison of volumes is only one way of defining the effective dimension, but it does lead to a reduced dimension

$$
D_{\text {eff }} \equiv D \frac{\ln \left(\mathbf{d}_{p}\right)}{\ln (\delta)}<D
$$

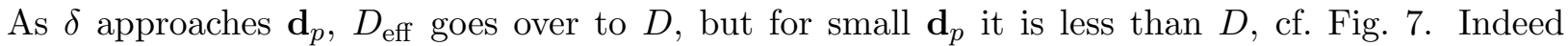
it becomes negative for $d_{p}<l_{c}$ (note that values $d_{p}<l_{c}$ are not included in the plot). This may appear surprising, but is a result of the randomness. Namely if $L=\left(\frac{V}{V_{c}}\right)^{\frac{1}{D}}$ is the length of the box in units of $l_{c}, \mathbf{d}_{p} \in(0, L)$ and cannot always be greater than $l_{c}$; indeed there is a small but non-vanishing probability that it is smaller. We must therefore view $D_{\text {eff }}$ more as a sign of dimensional reduction rather than interpret its value literally in the ultraviolet. Thus, dimensional reduction, present in many other approaches to quantum gravity, can in this sense also be seen in

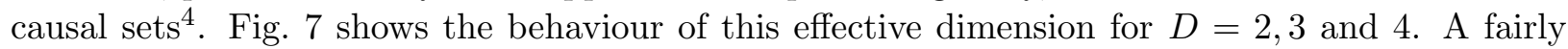
striking feature of this figure is that the convergence to the continuum occurs at $d_{p} \sim 10 l_{c}$ for all dimensions, which marks the scale of kinematic discretisation.

We conclude by bringing attention to another property of causal sets that could be connected to asymptotic silence ${ }^{5}$. Specifically, in an asymptotically silent spacetime, neighbouring worldlines do not meet, and so it becomes impossible for particles to scatter off each other. In causal sets, one might expect to observe a similar property for particle scattering at high center-of-mass-energies: A scattering event at center-of-mass-energy $M$ takes up a spacetime volume that decreases with increasing $M$. Accordingly, scattering events at center-of-mass-energies corresponding to the inverse cut-off scale should be expected to take place within a spacetime-interaction region of volume $V_{c}$. In a causal set, the probability that there is a causal set element within a given volume rapidly shrinks to zero as one reaches the cut-off scale. Therefore, in a causal set universe all particles become

\footnotetext{
${ }^{4}$ Note that this is not in contradiction to the increase of the spectral dimension in causal sets [29], as different dimensional estimators can exhibit different behaviour in the quantum gravity regime (see, e.g., 10]).

5 This idea is due to Rafael Sorkin.
} 
"transparent" to each other at very high center-of-mass energies, and scattering no longer takes place. It is interesting to observe that another approach to quantum gravity, namely the asymptotic safety scenario, exhibits hints of a similar behavior at high energies [68, 69, and in fact also exhibits signs of dimensional reduction which have been discussed in relation to asymptotic silence.

\section{Acknowledgments}

We thank Rafael Sorkin for discussions. We also thank David Rideout for help with Cactus. The simulations were performed using the Cactus Causal Set Toolkit [70]. A. E. is supported by the DFG under the Emmy-Noether program, grant no. EI-1037-1, and by an Emmy-Noether visiting fellowship at the Perimeter Institute for Theoretical Physics. Research at Perimeter Institute is supported by the Government of Canada through Industry Canada and by the Province of Ontario through the Ministry of Economic Development \& Innovation. S. S. was supported in part under an agreement with Theiss Research and funded by a grant from the FQXI Fund on the basis of proposal FQXi-RFP3-1346 to the Foundational Questions Institute.

[1] J. Ambjorn, J. Jurkiewicz and R. Loll, Phys. Rev. Lett. 95, 171301 (2005) doi:10.1103/PhysRevLett.95.171301 hep-th/0505113].

[2] D. Benedetti and J. Henson, Phys. Rev. D 80, 124036 (2009) doi:10.1103/PhysRevD.80.124036 arXiv:0911.0401 [hep-th]].

[3] C. Anderson, S. J. Carlip, J. H. Cooperman, P. Horava, R. K. Kommu and P. R. Zulkowski, Phys. Rev. D 85, 044027 (2012) doi:10.1103/PhysRevD.85.044027, 10.1103/PhysRevD.85.049904 arXiv:1111.6634 [hep-th]].

[4] A. Gorlich, arXiv:1111.6938 [hep-th].

[5] J. H. Cooperman, doi:10.1007/s10701-015-9972-8 arXiv:1410.0670 [gr-qc].

[6] J. Laiho, S. Bassler, D. Coumbe, D. Du and J. T. Neelakanta, arXiv:1604.02745 [hep-th].

[7] J. Laiho and D. Coumbe, Phys. Rev. Lett. 107, 161301 (2011) doi:10.1103/PhysRevLett.107.161301 arXiv:1104.5505 [hep-lat]].

[8] O. Lauscher and M. Reuter, JHEP 0510, 050 (2005) doi:10.1088/1126-6708/2005/10/050 hepth/0508202.

[9] O. Lauscher and M. Reuter, In *Fauser, B. (ed.) et al.: Quantum gravity* 293-313 hep-th/0511260.

[10] M. Reuter and F. Saueressig, JHEP 1112, 012 (2011) doi:10.1007/JHEP12(2011)012 arXiv:1110.5224 [hep-th]].

[11] S. Rechenberger and F. Saueressig, Phys. Rev. D 86, 024018 (2012) doi:10.1103/PhysRevD.86.024018 arXiv:1206.0657 [hep-th]].

[12] G. Calcagni, A. Eichhorn and F. Saueressig, Phys. Rev. D 87, no. 12, 124028 (2013) doi:10.1103/PhysRevD.87.124028 arXiv:1304.7247 [hep-th]].

[13] P. Horava, Phys. Rev. Lett. 102, 161301 (2009) doi:10.1103/PhysRevLett.102.161301 arXiv:0902.3657 [hep-th]].

[14] T. P. Sotiriou, M. Visser and S. Weinfurtner, Phys. Rev. Lett. 107, 131303 (2011) doi:10.1103/PhysRevLett.107.131303 [arXiv:1105.5646 [gr-qc]].

[15] G. Calcagni, Phys. Rev. E 87, no. 1, 012123 (2013) doi:10.1103/PhysRevE.87.012123 arXiv:1205.5046 [hep-th]].

[16] G. Amelino-Camelia, M. Arzano, G. Gubitosi and J. Magueijo, Phys. Lett. B 736, 317 (2014) doi:10.1016/j.physletb.2014.07.030 [arXiv:1311.3135 [gr-qc]].

[17] M. Arzano and T. Trzesniewski, Phys. Rev. D 89, no. 12, 124024 (2014) doi:10.1103/PhysRevD.89.124024 [arXiv:1404.4762 [hep-th]].

[18] N. Alkofer, F. Saueressig and O. Zanusso, Phys. Rev. D 91, no. 2, 025025 (2015) doi:10.1103/PhysRevD.91.025025 arXiv:1410.7999 [hep-th]]. 
[19] G. Calcagni, D. Oriti and J. Thürigen, Phys. Rev. D 91, no. 8, 084047 (2015) doi:10.1103/PhysRevD.91.084047 [arXiv:1412.8390 [hep-th]].

[20] M. Ronco, Adv. High Energy Phys. 2016, 9897051 (2016) doi:10.1155/2016/9897051 arXiv:1605.05979 [gr-qc]].

[21] M. Arzano and F. Nettel, Phys. Lett. B 767, 236 (2017) doi:10.1016/j.physletb.2017.02.005 arXiv:1611.10343 [hep-th]].

[22] S. Carlip, AIP Conf. Proc. 1196, 72 (2009) doi:10.1063/1.3284402 [arXiv:0909.3329 [gr-qc]].

[23] S. Carlip, arXiv:1009.1136 [gr-qc].

[24] S. Carlip, R. A. Mosna and J. P. M. Pitelli, Phys. Rev. Lett. 107, 021303 (2011) doi:10.1103/PhysRevLett.107.021303 [arXiv:1103.5993 [gr-qc]].

[25] S. Carlip, AIP Conf. Proc. 1483, 63 (2012) doi:10.1063/1.4756963 arXiv:1207.4503 [gr-qc]].

[26] S. Carlip, Int. J. Mod. Phys. D 25, no. 12, 1643003 (2016) doi:10.1142/S0218271816430033 arXiv:1605.05694 [gr-qc]].

[27] B. L. Hu and D. J. O'Connor, Phys. Rev. D 34, 2535 (1986). doi:10.1103/PhysRevD.34.2535

[28] J. M. Heinzle, C. Uggla and N. Rohr, Adv. Theor. Math. Phys. 13, no. 2, 293 (2009) doi:10.4310/ATMP.2009.v13.n2.a1 gr-qc/0702141.

[29] A. Eichhorn and S. Mizera, Class. Quant. Grav. 31, 125007 (2014) doi:10.1088/0264-9381/31/12/125007 arXiv:1311.2530 [gr-qc]].

[30] A. Belenchia, D. M. T. Benincasa, A. Marciano and L. Modesto, Phys. Rev. D 93, no. 4, 044017 (2016) doi:10.1103/PhysRevD.93.044017 [arXiv:1507.00330 [gr-qc]].

[31] S. Carlip, Class. Quant. Grav. 32, no. 23, 232001 (2015) doi:10.1088/0264-9381/32/23/232001 arXiv:1506.08775 [gr-qc]].

[32] S. Major, D. Rideout and S. Surya, Class. Quant. Grav. 26, 175008 (2009) doi:10.1088/02649381/26/17/175008 arXiv:0902.0434 [gr-qc]].

[33] S. Major, D. Rideout and S. Surya, J. Math. Phys. 48, 032501 (2007) doi:10.1063/1.2435599 grqc/0604124.

[34] F. Dowker, J. Henson and R. D. Sorkin, Mod. Phys. Lett. A 19, 1829 (2004) [gr-qc/0311055].

[35] L. Philpott, F. Dowker and R. D. Sorkin, Phys. Rev. D 79, 124047 (2009) arXiv:0810.5591 [gr-qc]].

[36] R. D. Sorkin, In *Oriti, D. (ed.): Approaches to quantum gravity* 26-43 [gr-qc/0703099 [GR-QC]].

[37] L. Bombelli, J. Lee, D. Meyer and R. Sorkin, Phys. Rev. Lett. 59, 521 (1987).

[38] J. Henson, arXiv:1003.5890 [gr-qc].

[39] F. Dowker, doi:10.1142/9789812700988-0016 gr-qc/0508109.

[40] R. D. Sorkin, doi:10.1007/0-387-24992-3-7 gr-qc/0309009.

[41] F. Dowker, Gen. Rel. Grav. 45, no. 9, 1651 (2013). doi:10.1007/s10714-013-1569-y

[42] S. W. Hawking, A. R. King and P. J. Mccarthy, J. Math. Phys. 17, 174 (1976). doi:10.1063/1.522874

[43] D. B. Malament, J. Math. Phys. 18, 1399-1404 (1977)

[44] J. Henson, D. P. Rideout, R. D. Sorkin and S. Surya, arXiv:1504.05902 [math.CO].

[45] A. Belenchia, D. M. T. Benincasa and S. Liberati, JHEP 1503, 036 (2015) doi:10.1007/JHEP03(2015)036 arXiv:1411.6513 [gr-qc]].

[46] M. Saravani and S. Aslanbeigi, Phys. Rev. D 92, no. 10, 103504 (2015) doi:10.1103/PhysRevD.92.103504 [arXiv:1502.01655 [hep-th]].

[47] A. Belenchia, D. M. T. Benincasa, S. Liberati, F. Marin, F. Marino and A. Ortolan, Phys. Rev. Lett. 116, no. 16, 161303 (2016) doi:10.1103/PhysRevLett.116.161303 [arXiv:1512.02083 [gr-qc]].

[48] R. D. Sorkin and Y. K. Yazdi, arXiv:1611.10281 [hep-th].

[49] N. Afshordi, M. Buck, F. Dowker, D. Rideout, R. D. Sorkin and Y. K. Yazdi, JHEP 1210, 088 (2012) doi:10.1007/JHEP10(2012)088 arXiv:1207.7101 [hep-th]].

[50] Rafael D. Sorkin. Spacetime and Causal Sets - World Scientific. in J.C. D'Olivo, E. Nahmad-Achar, M. Rosenbaum, M.P. Ryan, L.F. Urrutia and F. Zertuche (eds.) Relativity and Gravitation: Classical and Quantum (Proceedings of the SILARG VII Conference, held Cocoyoc, Mexico, December, 1990), pages 150-173 Singapore, 1991). http://128.230.72.124/ sorkin/some.papers/66.cocoyoc.pdf.

[51] R. D. Sorkin, Int. J. Theor. Phys. 36, 2759 (1997) doi:10.1007/BF02435709 [gr-qc/9706002].

[52] M. Ahmed, S. Dodelson, P. B. Greene and R. Sorkin, Phys. Rev. D 69, 103523 (2004) doi:10.1103/PhysRevD.69.103523 astro-ph/0209274.

[53] J. D. Barrow, Phys. Rev. D 75, 067301 (2007) doi:10.1103/PhysRevD.75.067301 gr-qc/0612128].

[54] N. Zwane, N. Afshordi and R. D. Sorkin, arXiv:1703.06265 [gr-qc]. 
[55] L. Bombelli, J. Henson and R. D. Sorkin, Mod. Phys. Lett. A 24, 2579 (2009) doi:10.1142/S0217732309031958 gr-qc/0605006.

[56] L. Glaser and S. Surya, Phys. Rev. D 88, no. 12, 124026 (2013) doi:10.1103/PhysRevD.88.124026 arXiv:1309.3403 [gr-qc]].

[57] J. Ambjorn and R. Loll, Nucl. Phys. B 536, 407 (1998) doi:10.1016/S0550-3213(98)00692-0 hepth/9805108.

[58] J. Ambjorn, A. Goerlich, J. Jurkiewicz and R. Loll, Phys. Rept. 519, 127 (2012) doi:10.1016/j.physrep.2012.03.007 arXiv:1203.3591 [hep-th]].

[59] J. Ambjorn, J. Jurkiewicz and R. Loll, In *Oriti, D. (ed.): Approaches to quantum gravity* 341-359 hep-th/0604212.

[60] J. Ambjorn, S. Jordan, J. Jurkiewicz and R. Loll, Phys. Rev. Lett. 107, 211303 (2011) doi:10.1103/PhysRevLett.107.211303 arXiv:1108.3932 [hep-th]].

[61] D. Kleitman and B. Rothschild, Proc. Amer. Math. Society 25, $276 ? 282$ (1970)

[62] D. P. Rideout and R. D. Sorkin, Phys. Rev. D 61, 024002 (2000) doi:10.1103/PhysRevD.61.024002 gr-qc/9904062.

[63] D. M. T. Benincasa and F. Dowker, Phys. Rev. Lett. 104, 181301 (2010) doi:10.1103/PhysRevLett.104.181301 [arXiv:1001.2725 [gr-qc]].

[64] D. M. T. Benincasa, F. Dowker and B. Schmitzer, Class. Quant. Grav. 28, 105018 (2011) doi:10.1088/0264-9381/28/10/105018 [arXiv:1011.5191 [gr-qc]].

[65] F. Dowker and L. Glaser, Class. Quant. Grav. 30, 195016 (2013) doi:10.1088/0264-9381/30/19/195016 arXiv:1305.2588 [gr-qc]].

[66] S. Surya, Class. Quant. Grav. 29, 132001 (2012) doi:10.1088/0264-9381/29/13/132001 arXiv:1110.6244 [gr-qc]].

[67] S. Major, D. Rideout and S. Surya, Class. Quant. Grav. 23 (2006) 4743 [gr-qc/0506133].

[68] D. F. Litim and T. Plehn, Phys. Rev. Lett. 100, 131301 (2008) doi:10.1103/PhysRevLett.100.131301 arXiv:0707.3983 [hep-ph]].

[69] B. Dobrich and A. Eichhorn, JHEP 1206, 156 (2012) doi:10.1007/JHEP06(2012)156 arXiv:1203.6366 [gr-qc]].

[70] Gabrielle Allen, Tom Goodale, Frank Löffler, David Rideout, Erik Schnetter, Eric L. Seidel, arXiv:1009.1341

[71] Y. K. Yazdi and A. Kempf, arXiv:1611.09947 [hep-th]. 